\title{
TAXONOMIA DE HYPHOMYCETES, UN TRIBUTO A NUESTRA PACIENCIA
}

Eduardo Piontelli L.

Universidad de Valparaiso Facultad de Medicina Càtedra de micologia Casilla 92 V Valparaiso

Palabras clave: Taxonomía fúngica, Hyphomycetes, Hongos anamórficos.

Key words: Fungal taxonomy, Hyphomycetes, Anamorphic fungi.

\section{RESUMEN}

Se analiza y comenta la problemática actual existente en la clasificación de los Hyphomycetes, donde aún predomina el heterogéneo criterio personal del taxónomo feneticista, abarcando los siguientes temas tales como: concepto de especie, taxa infraespecíficos, conidiogénesis, conidióforo, conidios, conidiomata, pleoanamorismo, etc.

Se concluye con un enfoque moderno que destaca la frecuente aplicación en la literatura de las nuevas herramientas taxonómicas, en especial, la biologia molecular a nivel de secuencias de DNA y RNA, Situación que ayudará con seguridad a dilucidar en el futuro las relaciones filogenéticas de estos hongos.

\section{SUMMARY}

[Hyphomycetes taxonomy, a tribute to our patience]

It is analyzed and commented the real problematic existing in the classification of the Hyphomycetes where there still predominates the personal heterogeneous criteria of the pheneticist taxonomist, such as: species concept, infraspecific taxa, conidiogenesis, conidiophores, conidia, conidiomata, pleoanamorphism, and so on.

It is concluded with a modern approach that enphasized the frecuent application in the literature of the new taxonomic tool, specially molecular biology in relation to DNA and $R N A$ sequence, all this will surely help to elucidate in the future phylogenetic relations in these fungi.

de DNA nuclear, ribosomal y mitocondrial, la quimiotaxonomía, la ultraestructura, la serología, los patrones enzimàticos, la taxonomía numèrica, etc. (Minter 1987, Romero \& Minter 1988, Mugnai et al. 1989 , Monte et al. 1990, Arambarri \& Cabello 1989, Frisvald et al. 1990, Samson \& Pitt 1990, Cavalier-Smith 1989, Bruns et al. 1989).

Estas nuevas tendencias, no siempre resultan ser ideales, pueden ser difíciles de aceptar por la mayoría de los micologos, o exhiben aùn muchos argumentos controversiales, metodoldgicos, tedricos o interpretativos, que necesariamente deberàn evaluarse con prudencia en el tiempo y con mayor experimentación. A veces somos injustos con el taxónomo profesional, este particular e introvertido personaje que a pesar de poseer todos los defectos y virtudes propias de su especie, està cumpliendo dentro de sus posibilidades una gran ambición, como el tratar de ordenar el curso evolutivo de un determinado grupo de organismos, situacion que no siempre resulta transparente al lector.

En estos días, existe la sensaciòn que los antiguos esquemas morfologgicos feneticistas, estàn relegados a un segundo plano frente a los nuevos caminos biológicos, fisiologicos y bioquìmicos. Pero a pesar de los nuevos avances, la metodología general 


\section{CUADRO 1. CONCEPTO DE ESPECIE}

Unidades genéticas homogéneas

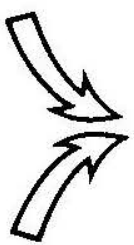

Unidad taxonómica básica

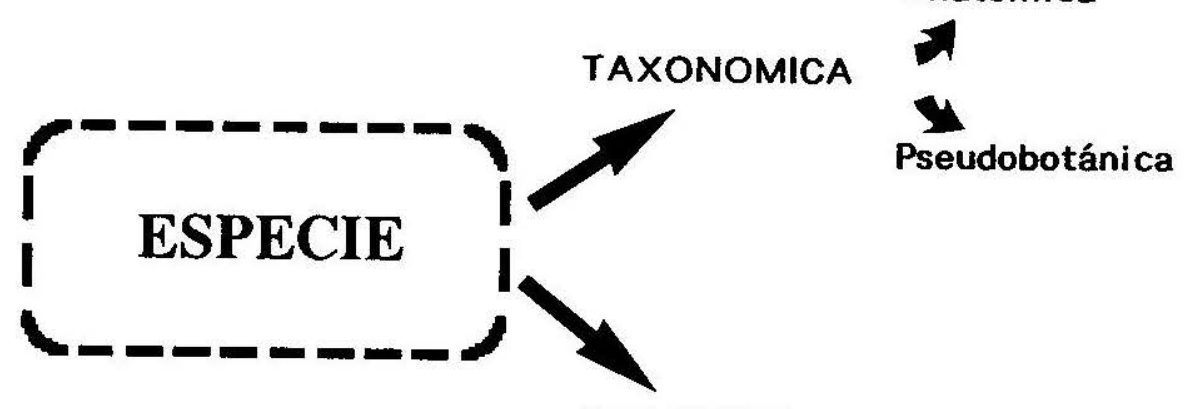

Anatómica

BIOLOGICA

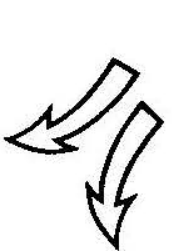

$\sqrt{4}$

Grupos de cruzamiento

Características filogenéticas (Especie molecular)

Características fenéticas

( Morfo o fisioespecie)

Características evolutivo/ecológicas

(Especiación ecológica,aislamiento geográfico)

\section{CUADRO 2. TAXA INFRAESPECIFICOS}

Aislamiento geográfico

\section{SUB-ESPECIE}

Sub-poblaciones cont diferencias y menos similitudes

RAZA

RAZA FISIOLOGICA

Idénticos set de locus de virulencia. Sub-poblaciones con + similitudes y menos diferencias
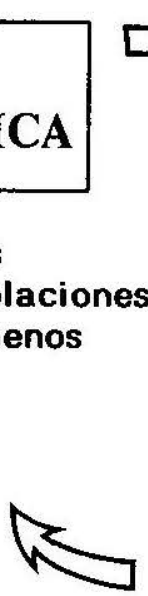

Cualquier variación morfológica menor

P A TO T I P O

(?)
Mayores cruzamientos entre las sub-especies que con la especie<smiles>C1=CCCC1</smiles>

Sub-poblaciones polimórficas (Genetistas,biólogos de poblacio-) nes

\section{Forma specialis}

Parasitismo sobre hospedador específico 
de identificación morfologica y fisiologica en uso no podrá cambiarse seguramente hasta las primeras décadas del nuevo siglo... La razón es muy simple: según Pitt \& Samson (1989), la taxonomía a nivel genérico debe ser accesible tanto en Santiago, Buenos Aires, Nueva York o París, como también para aquellos que trabajan en los centros especializados de Berlín, Kew o Baarn. Sin embargo, el reunir aislamientos fúngicos en series lógicas de grupos constituídos por géneros, especies o sub-especies (bajo el Código Internacional de Nomenclatura Botánica CINB), tiene límites a veces difíciles de definir y en muchos casos la distinción entre estos taxa, puede depender de un pequeño número de criterios taxonómicos o a veces por una simple diferencia (Pitt \& Hocking 1985), lo que se transforma en situaciones de límites o formas que escapan fácilmente al no experto.

Si consideramos a la taxonomía como un conjunto de reglas y mètodos que permiten reunir a los individuos en grupos homogèneos, mediante un set de atributos físicos o fisiologicos, la selección de èstos debe efectuarse actualmente, por todos los metodos aconsejables o disponibles. De esta forma el resultado serà una clasificacionn cada vez menos artificial.

\section{CONCEPTO DE ESPECIE Y TAXA INFRAESPECIFICOS}

La especie,es el nivel taxonómico básico en la identificación biológica,sin embargo, su definición es aún una situaciòn conflictiva, que generalmente tratamos de evadir. Idealmente, hablar de especie, es referirse a grupos de organismos capaces de cruzarse entre si y producir descendientes ferrtiles, pero su concepto moderno es aùn màs amplio, e incluye por lo menos 2 tipos de categorías bassicas: una Taxonómica, usada principalmente por los morfologos y fisiologos y otra Biologica, usada por genetistas y biologos de poblaciones. Sin embargo, la literatura ortodoxa mantiene vigentes un sinnumero de conceptos distintos, acorde a la metddica seguida en la determinación de similitudes o diferencias (Morfoespecie, Paleoespecie, Coenoespecie, Agamoespecie, etc).

La amplia variacion morfologica que puede existir en organismos con el mismo stock genético (clina), dentro de los miembros (individuos) de una poblaciòn, ha llevado a los investigadores a considerar más seriamente el concepto bioldgico de especie, que el taxonómico, debido a que este ùltimo refleja en mayor o menor medida la experiencia, o la opinion personal del taxdnomo. No es facil aseverar y determinar las semejanzas o las pequeñas variaciones de un individuo dentro de una poblacion, y el taxònomo al imponer su criterio personal en favor de una de estas situaciones feneticas, puede ofrecer resultados a veces polèmicos para otros taxònomos. Desde este punto de vista y a la luz de las nuevas evidencias, el clasico concepto bioldgico de especie resulta empírico y teoricamente inadecuado. Actualmente los especialistas en especiacion lo evaluan desde 4 categorias: grupos de cruzamiento, fenéticas, evolutivo/ecológicas y filogenèticas. Solo la vision filogenètica de la especie, emerge con la mayor claridad conceptual, especialmente cuando se asocia a programas empiricos de estudios de especiación, los cuales pueden iniciarse con un detallado anàlisis cladistico (Minsler 1991) (Cuadro 1).

En general los micologos de diferentes especialidades microbiológicas, no tienen una marcada tendencia en estudiar las poblaciones füngicas en conjunto, normalmente se analiza un solo aislamiento obtenido del ambiente para su posterior inclusion en un taxon (o muchas veces la descripciòn de uno nuevo). Con esto se reduce la apreciación de la vitalidad y plasticidad del conjunto de individuos semejantes que constituyen una población determinada, situación que puede subsanarse al examinar en el tiempo un mayor nùmero de individuos. Esta labor debiera ser efectuada por los micologos de alimentos, micologos mèdicos, fitopatologos, taxònomos, genetistas, etc.. De esta manera es posible definir las unidades genèticas homogèneas, considerando la variacion fenetica, barreras reproductivas, especiaciòn ecoldggica, aislamiento geogràfico, etc., con una positiva apreciación de los continuos y discontinuos cambios dentro y entre las poblaciones, en aras de una taxonomía más estable.

Existen ademàs problemas interpretativos a nivel de la terminología nomenclatural actual, que por su escasa flexibilidad, no permite unificar aùn bajo el nivel de especie, los conceptos jerarquicos de las unidades poblacionales (Sub-especie, Raza, Genet, Ramet, etc.), con las no jeràrquicas (Biotipos), empleados en las distintas disciplinas relacionadas con la micologia. Mucha de esta tradicional terminologia, es a veces ambigua, mal definida o problematica en su empleo para el micdlogo. Brasier y Rayner (1987), discuten esta situacion para los hongos, refiriendose a los tèrminos de: Genet, Ramet, Raza, Forma, Variedad, Sub-especie, u otra expresión que permita cubrir distintas y divergentes sub-poblaciones que no tienen en general un total aislamiento reproductivo y que exhiben regularmente un orden de diferencias para una variedad de caracteres morfofisioldgicos.

Para nosotros el aislamiento, es el primer cultivo de un determinado hongo desde un ambiente particular, èste se transforma en una cepa (strain), cuando ha sido multiplicada asexualmente y mantenida en cultivo. Esta cepa es un Genet o una unidad - colección genética discreta, del cual deriva por propagacion asexual vegetativa, un Ramet. En las 


\section{CUADRO 3. VARIACION}

Cambios graduales en las formas (formas intermedias)

\section{CONTINUA
(Poligenes) \\ INFLUENCIAS AMBIENTALES}

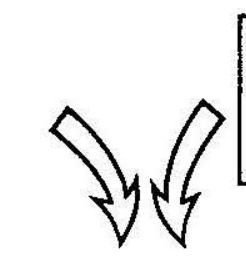

Cambios en el tiempo sin formas intermedias

\section{DISCONTINUA \\ (Pocos o un solo ) \\ gen mayor}

\section{DIFERENCIAS}

ALELICAS

(previene la he-

terocariosis)

$\checkmark$

INCOMPATIBILIDAD

VEGETATIVA

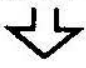

Factores nutricionales, edad,climáticos,etc.

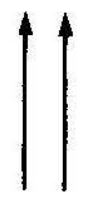

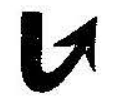
Variacion

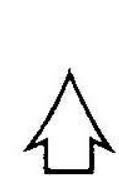

DIFERENCIAS EN LAS CARACTERISTICAS MORFO-FISIOLOGICAS ENTRE LOS MIENBROS DE UNA POBLACION

\section{CONSTITUCION GENETICA}

\section{(Genotipo)}

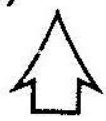

Recombinación en la meiosis, parasexualidad. MUTACION

\section{CUADRO 4. CONCEPTO DE ESPECIE EN HONGOS ANAMORFICOS*}<smiles>C1CC2CC12</smiles>

Se basa en un reducido número de caracteres en géneros con pocas especies

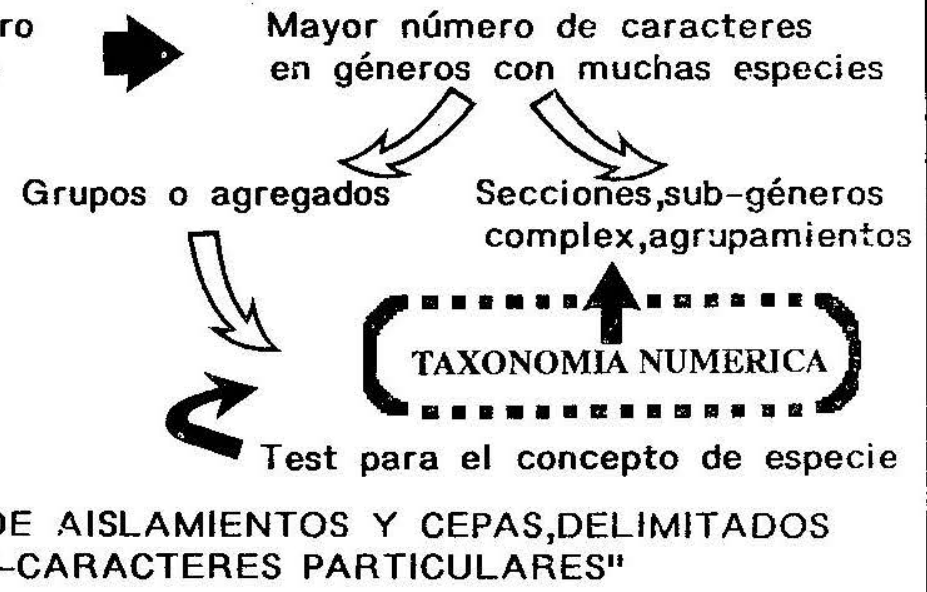

Mayor número de caracteres géneros con muchas especies
Secciones,sub-géneros complex,agrupamientos 
Razas, las similitudes exhibidas por esta sub-población, pueden pesar màs que sus diferencias (lo contrario sucede en la Sub-especie). El tèrmino de Raza o Raza fisiológica, es usado corrientemente en diferentes sentidos por los fitopatòlogos (Caten 1987), pero preferentemente significa la presencia del mismo set de locus de virulencia (debiera reemplazarse por patotipo, sensu Robinson (1969)), sin embargo, los genetistas, ecologos y biologos de poblaciones lo emplean para la descripcion de sub-poblaciones polimorficas dentro de las especies. El tèrmino de raza, se usa también para indicar grupos ètnicos o religiosos y para muchos es preferible referirse a subespecie. La Variedad, otro tèrmino bastante liberal y muy empleado en las formas cultivadas de plantas (cultivar), tiene el mismo significado dentro de las especies, como así el de forma. (Cuadro 2)

Las sub-especies, tienen características en comùn y tienden a cruzarse entre ellas, màs que con los otros miembros de la especie, usualmente debido al aislamiento geogràfico.

El tèrmino comùn de cepa, se usa indiscriminadamente para definir cualquier tipo de aislamiento con un genotipo específico, desde una mayor a menor sub-unidad de la especie, asì como el de variedad y forma (excluyendo de este ultimo el de forma speciales).

Como si fuera poco esta "semàntica literaria" no termina aquì cuando se emplean en forma subordinada o correlativa, la nomenclatura de los biotipos, para designar un grupo de "cepas" con caracteristicas comunes. Este sistema es conceptualmente semejante al Codigo Internacional de Nomenclatura Bacteriologica o el de "Deme", empleado por algunos taxonomos vegetales. La amplia variedad de biotipos ( eco, gamo, morfo, pato, sero, cariotipo, etc.), guarda relación directa o indirecta con las distintas jerarquías taxonòmicas en uso bajo el nivel de especie.

\section{CLASIFICACION DE LOS HONGOS ANAMORFICOS}

¿Què sucede en los hongos anamorficos o con ciclos mayoritariamente asexuados, donde el concepto de especie biologica no puede aplicarse en todo su sentido?. Normalmente nos referimos a ellos como un reducido grupo de aislamientos y cepas delimitadas por uno o màs estados-caracteres particulares, " donde la transicion entre tales grupos, - puede ser clara o vaga y la brecha entre las especies puede encontrarse a varios niveles de disimilitud " (De Hoog \& Smith 1984).

El problema se presenta en forma seria en los momentos que nuestra labor de micologo se radica en la identificaciòn y ubicaciòn de un aislamiento o cepa, en una especie dentro de un gènero bien definido; èsto es relativamente simple en aquellos con un reducido nùmero de especies. En estos casos el concepto de especie se basa generalmente en un pequeño nùmero de caracteres, pero la situaciòn es diametralmente opuesta en los que presentan un gran nùmero de èstas. Los ejemplos en la actualidad serian innumerables, por ejemplo: Colletotrichum, Phoma, Phomopsis, Penicillium, Aspergillus, Fusarium, Chrysosporium, Trichophyton, etc., los cuales en el pasado se han circunscrito en Grupos, Agregados, Complex, u otro calificativo de poco valor taxonòmico. En la actualidad es preferible delimitarlos como Secciones y Sub-gèneros.

Es en estos taxa u otros, donde las delimitaciones genèricas o intraespecíficas pueden derivar de distintos conceptos adoptados (otro problema en la literatura) mediante el empleo de características consideradas poco naturales y donde los intentos morfologicos, ontogènicos y las conexiones teleomòrficas no han podido entregar aùn suficientes aportes para la sistemática. Es aqui donde la literatura nos muestra la utilidad de interrelacionar los paràmetros morfo-fisioldgico con los bioquímicos u otros, mediante estudios multidisciplinarios, con herramientas tales como el analisis aportado por la Taxonomía numèrica (Bridge et al. 1989; Monte et al. 1990; Mugnay et al. 1989), la cual actùa al mismo tiempo como un test para el concepto de especie. Esta herramienta, sin embargo, no se emplea en forma rutinaria en el laboratorio (Cuadro 3). Frente a estas dificultades, optamos generalmente por abandonar en un rincòn del laboratorio la cepa difícil para un futuro anàlisis, que normalmente no se efectuarà, aludiendo que tal o cual gènero es demasiado complicado o heterogèneo, a pesar de contar en nuestros archivos con buenas monografías. La problemàtica taxonòmica de los Hyphomycetes, radica en el manejo personal de ciertas situaciones o en la aplicaciòn de criterios determinativos genèricos, relacionados principalmente con ciertos caracteres morfoldgicos o físiologicos aplicados en la clasificacion, algunos de los cuales pueden condensarse en los siguientes puntos:

\section{1.- PLEOMORFISMO}

El pleomorfismo (sensu Du Bary), incluye las implicaciones de la variación a nivel de las estructuras empleadas con fines taxonomicos. Basicamente consiste en que un hongo sea capaz de expresarse en sus 2 formas (una sexual y otra asexual), o que los teleomorfos estrechamente relacionados puedan tener diferentes anamorfos o viceversa. Este es el caso de Penicillium, Aspergillus, Fusarium, Nectria, Arthroderma, Gliocladium, etc., situacion que dificulta la comprensión de sus fílogènias, sin excluir la posibilidad de que algunos gèneros anamorfos puedan ser filogenèticamente homogèneos, aún correspondiendo 
a distintos teleomorfos. Los casos de estrecha relaciòn, permiten un dptimo concepto de especie, existiendo buenos ejemplos como: Geotrichum-Dipodascus, Chloridium-Chaetosphaeria, TubeufiaHelicosporum, etc.

Al parecer la presión evolutiva ha operado practicamente en forma independiente en cada ciclo de vida, lo cual no debe sorprendernos, si consideramos los distintos roles desempeñados por anamorfos y teleomorfos. "Las necesidades ecologicas parecen haber llevado a un efectivo enmascaramiento de las similitudes genèticas" (Di Cosmo et al. 1983), y como los teleomorfos de muchos hongos anamòrficos nose conocen en la actualidad, nuestra aproximación a la clasificaciòn puede ser netamente pragmàtica. (Halubovà-Jechovà 1990).

\section{2.- VARIACION}

La variación consiste en las diferencias de las mùltiples características ya sea morfològicas como genèticas entre los individuos de una poblaciòn. Esto se debe a: influencias ambientales, a la constituciòn genètica del individuo (genotipo), o la interacciòn entre ambas. La variación genètica es debida a la recombinaciòn de genes durante la reproducciòn sexual y la mutaciòn; esta ùltima es tambièn importante en los hongos anamòrficos, junto a la heterocariosis y la parasexualidad. Algunas especies tienen una amplia variacion genètica como es el caso de Aspergillus nidulans (= A.nidulellus?), el cual contiene 19 grupos de compatibilidad de heterocarión (incompatibilidad vegetativa), bajo control heterogénico, con una diferencia alelica en cada uno de por lo menos 8 Het genes específicos suficiente para prevenir la formaciòn de heterocariones entre un par de cepas (Brasier 1987, Croft 1987). Es por esto que la incompatibilidad vegetativa delimita entonces el concepto de genoespecie. La variación puede ser continua, donde intervienen varios genes (Poligenes), cada uno con poco efecto, causando cambios graduales en las características de la especie, o discontinua, donde intervienen pocos o un solo gen mayor que pueden o puede estar presente en 20 más formas alélicas, originando características especiales en el fenotipo, lo que hace diferir una especie de otra sin formas intermedias entre ellas. En la literatura frecuentemente encontramos términos como variación molecular (evolutiva), variación fenética y genética.

Los grados de variación morfológica son difíciles de apreciar o interpretar, debido a que las similitudes o diferencias micro y macroscópicas de las cepas estudiadas, pueden expresarse en varios niveles del fenotipo. Bridge et al (1986), al analizar las variaciones a nivel morfológico y bioquímico en 3 especies de Penicillium a partir de las siembras de un solo conidio, pudieron comprobar que éstos pueden dar origen principalmente a 2 tipos de colonias es- tables (Tipo W y R), además de otro tipo inestable (menos frecuente), llamado del Tipo $\mathbf{O}$ (observado en P. viridicatum). Este ultimo presenta un bajo porcentajes de conidios grandes, anormales y uninucleados (seguramente poliplóides). Sin embargo la progenie del tipo $O$, muestra propiedades en común con las 2 líneas estables.

Una explicación posible de estas variaciones morfo fisiológicas y bioquímicas es el ciclo parasexual. El tipo $R$, con una presencia de un $44 \%$ en cultivo, puede corresponder a una variante morfológica debida a los subcultivos y con desventajas ecologicas-competitivas frente al tipo $\mathbf{W}$, con la misma presencia en cultivos y más adaptado al ambiente. Es por esto que las cepas tipo deberían guardarse desecadas. Como esta situación ha sido observada en otros Hyphomycetes, tales como Aspergillus y Cladosporium, entre otros (Prasil \& De Hoog 1988, Morgan-Jones y Jacobsen 1988), es recomendable antes de la descripción de una nueva cepa en cultivo, analizar su grado de variabilidad desde subcultivos obtenidos por monoconidios, de esta manera no se afectarían las conclusiones taxonómicas con posterioridad..

\section{3.- CONIDIOGENESIS}

A pesar de la gran información generada después del trabajo de Huges (1953-58), que permitió un alejamiento (aunque no total) del sistema Saccardiano, los criterios ontogénicos no pueden ser aplicados en términos absolutos y la conidiogénesis debe ser considerada como una función temporal (Minter et al. 1982). Algunos géneros tienen especies con diferentes desarrollos de células conidiógenas y ontogenia conidial (pleomorfismo). Las estructuras morfologicas producidas por los hongos pueden tener diferentes funciones y las resultantes conexiones entre estructuras-funciones, están bajo control genético. Estas expresiones pueden ser afectadas por las condiciones ambientales y la edad del cultivo; solo controlando estas variables puede obtenerse una información completa al respecto (Monte et al. 1990).

No es posible condensar en pocas líneas todo lo referente a conidiogénesis, es por éso que solo me referiré a la "fialídica" (la más común en los Deuteromycetes), esta célula conidiógena presenta tipicamente en el ápice un conspicuo engrosamiento periclinal, el collarete puede o no extenderse hacia los lados, pero raramente se aprecia como un embudo (Phialophora). En muchos casos el ápice es reducido, minuto, siempre evidente o ausente; este engrosamiento permite relacionar la ontogenia conidial como enteroblástica (formación de muchos conidios), y su falta como holoblástica (pocos o un solo conidio). Gams (1973), considera que una célula conidiógena que da origen a un solo conidio puede considerarse también como fílide.

Las fiálides, pueden presentar distintas forma, grosor y color; existiendo a veces relaciòn entre 
grupos de especies teleomorfas y la morfologia fialídica en sus anamorfos. Existen células conidiógenas monofialfdicas, (un solo locus conidiogeno), otras son polifialídicas (más de un locus por proliferación percurrente), como en Fusarium, Cylindrocarpon y bidimostilbe. Estos 3 géneros pueden presentar ambos tipos de estructuras. La formación de células poliblasticas por proliferación simpodial, es muy escasa en los Hyphomycetes, sin embargo Fusarium, Cladobotryum y Chaetopsina, la presentan, considerandose como un tipo de conidiogénesis holoblástica.

La producción de distintos tipos de físlides (mono-poli-poliblasticas), se observa en algunas especies de Fusarium y tiene cierto valor taxonómico a nivel de especie (Pascoe 1990a y b). Todas estas estructuras son dificiles de observar en cultivos viejos, más aún cuando estas desaparecen por lisis enzimática, no pudiendo apreciarse sobre los conidioforos en medios de cultivos viejos.

Todas las etapas relacionadas con la ontogenia y posterior liberación y regeneración del conidio ( proliferación, ontogenia, delimitación, secesión y regeneración Minter et al. 1982-83), permiten buenos aportes a nivel genérico. Si estas caracteristicas se relacionan con las anatómicas, químicas, serologicas u otras, tendremos en el tiempo una mejor delimitación de los géneros anamorfos.

\section{4.- CONIDIOFORO}

Los patrones de ramificación y disposición de los conidióforos relacionados con el tipo de conidiogénesis, el tamaño de la célula conidiógena y su posición en el estipe, son estructuras útiles en la separación de los géneros, a pesar que en todos ellos la ramificación no tiene el mismo valor. La terminolgias empleada para la ramificación del conidióforo no es consistente en la literatura, salvo en algunos géneros (Pitt 1979, Gams 1971). Seifert (1985), describe la terminología utilizada en la descripción de las ramificaciones de los conidióforos del género Stilbella e Hyphomycetes relacionados. Este autor incluye categorías tales como: monoclasial, donde un eje da origen a solo 2 ramas laterales (una vez monoclasial); si la ramificación se repite en el mismo eje, encontrandose a 2 niveles se considera 2 veces monoclasial; monoverticilados, terverticilados, 2 niveles de monoclasial, 2 niveles de verticilados y aclopleurógeno. Sin embargo no siempre es fácíl describir las ramificaciones del conidióforo, especialmente cuando ciertos patrones de ramificación están entremezćlados: situación que ha generado cierta divergencia entre los taxónomos para considerarlos en la delimitación a nivel genérico. Este es el caso de la inclusión de Cylindrotrichumm en Chaetopsis, por Di Cosmo et al.(1983), situación no aceptada por Halubova-Jechová (1990), Cabello y Arambarri (1988) y Arambarri y Cabello (1989), los cuales consideraron que las estructuras del conidióforo son diferentes. Algunos autores, aceptan los caracteres del conidióforo y la célula conidiógena como de importancia genérica, otros no aceptan como status genérico a los caracteres del conidio y los cambios de color de la pared del conidióforo en ácido láctico o KOH (Samuels 1985, Kirk \& Sutton 1985, Seifert 1985). Por todo lo expuesto, el sólo análisis de la ramificación del conidióforo, parece no tener un valor aún estable en la delimitación genérica salvo en algunos casos.

En los Hyphomycetes, encontramos 3 tipos básicos de patrones de ramificación del conidióforo; no ramificados, ramificados verticiladamente y ramiffcados peniciladamente (el Ǵltimo es el más frecuente). Como estos patrones son comunes en grupos no relacionados de anamorfos, deben emplearse otros caracteres morfológicos y la pigmentación. Cuando los géneros de anamorfos están ligados a varios teleomorfos no relacionados, los grupos de especies existentes indican que deben efectuarse subdivisiones de éstos (Samuels \& Seifert 1987).

Por ejemplo, en el género Acremonium, las especies que poseen conidióforos monofialídicos están relacionadas a teleomorfos en Hypocreales y otros taxa no relacionados, esta disposición tiene poco significado filogenético. Por ésto, los heterogéneos géneros de Acremonium y Phialophora (Gams \& McGinnis 1983), no siempre se delimitan fácilmente. El primero, posee teleomorfos asociados a 2 familias de Eurotiales (Cephalothecaceae y Pseudoeurotiaceae), como también a Chaetomiaceae, Clavicipitaceae y otros taxa (Gams \& McGinnis 1983). Mientras Phialophora, los presenta en Eurotiales, Lasiosphaeriaceae y Diaporthaceae. Debido a las pequeñas diferenciaciones y las dispersas relaciones de sus teleomorfos, se habla de especies agregadas (Art. 59 CINB).

Phialemonium, un género semejante a los dos mencionados con anterioridad, se delimita de sus anamorfos similares fialídicos, por sus cortos conidióforos (adelofiálides), o fiálides sin septo basal, o sea conidióforos intercalares sin engrosamiento periclinal y ausentes de pigmentación. Las adelofiálides son típicas y las fiálides discretas y largas son escasas. En Phialemontum, es importante la observación directa de los cultivos para visualizar sus falsas cabezas conidiales dispuestas en cortos conidioforos.

Lecytophora (=Margarinomyces), es derivada de Phialophora (?), por poseer adelofiálides semejantes a Phialemonium, pero con un ápice más ancho, engrosamiento periclinal y un corto collarete (Phialophora lignicola se trasladó por lo expuesto a Lecytophora lignicola). En Acremonium strictum, las adelofiálides son muy comunes y se producen cuando la hifa está sumergida, situación que también se observa en la sección Albo-Lanosa de Acremonium 
(A.coenophialum, A.typhinum , Morgan-Jones \& Gams 1982) .

Todas estas semejanzas o diferencias basadas solamente en la morfología, son fuertemente especulativas cuando no existe una indiscutible relación anamorfo-teleomorfo (La semejanza morfologica no siempre indica una estrecha semejanza genética).

Las especies del género Verticillium o semejantes, se relacionan a distintos grupos de Hypocreales, lo mismo sucede con Gliocladium (=Clonostachys), donde las ramificaciones peniciladas se presentan en los 3 mayores grupos de teleomorfos de Hypocreales (Hypomyces, Nectria e Hypocrea). La definición de este género ha sido expandida por Seifert (1985), para incluir las especies sinnematosas.

\section{5.- CONIDIOMATA}

Dentro de un determinado Orden o Familia, los anamorfos pueden tener una amplia variedad de formas (Rango de anamorfos), situación que se observa en Dothideales, Eurotiales, Hypocreales, etc. Si consideramos como ejemplo a estos últimos (Samuels \& Seifert 1987), veremos que existen aproximadamente más de 40 anamorfos relacionados, los cuales incluyen géneros de Coelomycetes e Hyphomycetes; entre éstos existe un amplio rango de formas en las estructuras productoras de conidios. (pleomorfismo)

La forma y grado de agregación de los conidioforos en los Hyphomycetes, es uno de los criterios importantes en su clasificación, tales como : esporodoquios, picnidlos y sinnemas. Sin embargo despues de Hughes (1953), los conidiomata, fueron relegados a caracteres secundarios en la taxonomía de los anamorfos. Todos los conidiomata, son derivados de un estroma, aunque no siempre es posible observarlo en cultivo. Los esporodoquios son poco frecuentes en las dematiáceas (pero constantes en algunos géneros). La presencia o ausencia del sinnema, también es considerada como un carácter de importancia secundario, ya que puede aparecer entre las especies que producen conidióforos laxos. Sin embargo, el análisis de la anatomía del sinnema, combinado con otros aspectos morfológicos como la ontogenia conidial, la textura de las masas conidiales y su forma, los patrones de ramificación, el tipo de desarrollo en cultivo u otros, pueden aportar información de las posibles relaciones entre ellos (Seifert \& Okada 1990).

Seifert (1985), propone una terminología descriptiva en relación al sinnema, sin descartar las posibilidades de categorías intermedias en éste, como también en otros tipos de conidiomata, cuando uno considerara el entero espectro de los hongos anamórficos. Varios géneros incluyen especies mono y sinnematosas, como Penicillium, Aspergillus, Hirsutella, pero también Doratomyces y Scopulariopsis. El caso particular de estos dos géneros, indicaría que deberían unificarse en uno sólo, situación que se respalda por sus estrechas relaciones teleomórficas. Otros géneros solo presentan especies sinnematosas solamente(Graphium), situación que se emplea como un importante carácter taxonómico. La formación del sinnema, es más frecuente en los hongos dematiáceos, especialmente en áreas tropicales y subtropicales; en este sentido se ha especulado sobre la influencia del clima en la agregación de los conidióforos. Si así fuere, habría limitaciones en emplear su presencia como criterio genérico (Halubová-Jechová 1990) No debe olvidarse que el comportamiento de los conidiomata es muy variable en cultivo, situación no muy útil para los fines taxonómicos.Sin embargo algunas especies producen estructuras típicas y estables en los cultivos y su estudio morfo-anatómico puede aportar en reconocer las relaciones o el rumbo evolutivo. Algunos ciclos de vida de un anamorfo, conservan la anatomía ancestral del teleomorfo. Varias especies de Trichophyton y Microsporum, pueden formar pseudo - cleistotecios rodeados por hifas peridiales características de su teleomorfo(Arthroderma), pero en su interior no contienen ascos sino abundantes microconidios (Okoshi \& Hasegawa 1966, Stockdale 1961,Saito et al. 1991).Esta situación la hemos observado también en Trichophyton terrestre complex en aislamientos de suelos chilenos(datos no publicados).

La importancia de los posibles cambios del pH en los pigmentos estromáticos y periteciales de algunos teleomorfos, ha sido enfatizado en la taxonomía de algunos Ordenes ( Dorenbosch 1970, Samuels 1976, Rossman 1983, Seifert y Samson 1985,). Un especímen es considerado $\mathrm{KOH}$ positivo, si el pigmento cambia de color cuando se incluye en $\mathrm{KOH}$ al $2 \%$ (desde algún grado de color amarillo, naranja, rojo-café a rojo-sangre, a púrpura,revertiendo a su color normal en ácido láctico al $90 \%$ ). Generalmente la reacción en $\mathrm{KOH}$ en los teleomorfos, está correlacionada con la que presentan los anamorfos asociados.

La presencia o ausencia de estroma, se emplea en las claves como ayuda genérica, sin embargo en algunas oportunidades un género puede incluir especies estromáticas o no. Aparentemente el grosor del estroma es también influenciado por las condiciones ambientales. Las formas estromáticas se presentan a menudo en sustratos de larga duración, donde la conservación del agua puede ser más importante que un desarrollo rápido en el ambiente. Generalmente, los teleomorfos estromáticos tiende a formar anamórfos estromáticos; estos últimos pueden asumir in vitro morfologías muy diferentes a las expresadas in vivo o en la naturaleza, incluso el grosor del estroma parece ser influenciado por las condiciones ambientales (Holubová-Jechová 1990). La presencia o ausencia de setas en los conidiomata picnidales, es otro carácter que se emplea en la delimitación genérica (Phoma,Pyrenochaeta), pero 
no siempre es útil (Van Der Aa et al. 1990). La textura y los caracteres de cultivo de los géneros productores de picnidios, se emplean constantemente, pero las especies no pueden ser identificadas correctamente usando solamente características morfologicas como en el género Phoma (Monte et al. 1990).

El conocimiento incompleto o insuficiente en la actualidad de los ciclos de vida, debido a la variabilidad de algunos teleomorfos (Ej. Phomopsis), nos encamina al empleo de métodos experimentales modernos a nivel molecular y genético, los cuales serán con seguridad las futuras necesidades.

\section{6.- CONIDIOS}

La morfología conidial y la septación son los elementos importantes en la distinción de las especies de Hyphomycetes, sin embargo, estos caracteres no deben ser sobreevaluados, debido a que a veces los géneros incluyen especies septadas y aseptadas (Chaetopsina), lo mismo sucede con el tamaño de éstos (Pitomyces, Endophragmiella,Monodyctis). El color claro u oscuro (Chalara,Chloridium), o como los conidios secos y húmedos de los Acremonium sección. Gliomastix, o el número de septos o tipo de éstos, ya sea los muriformes y multiseptados de Chalara y Endophragmiella.

Algunos taxa presentan características poco comunes, como las células basales pediceladas de los macrocroconidios de Fusarium, que permite delimitar algunas secciones (Booth 1971).

Los conidios de los anamorfos de Hypocreales, nacen usualmente o en gotas de liquido claro (Acremonium, Verticillium, Gliocladium penicillioides), u opaco (Tubercularia, Fusarium, Gliocladium roseum).

Varios hongos anamórficos tienen un bajo nivel de organización y complejidad, sus propágulos son poco differenciados de sus estructuras hifales, tal es el caso de las clamidosporas, la conidiogenesis simpodial (Tritirachium, Acrodontium, Beauveria), o la conidiogénesis artrica (Geotrichum), o es de tipo levaduriforme.Algunos géneros contienen especies fuertemente pleoanamórficas, a veces no relacionadas o de dudosa identidad, pueden presentar similitudes en sus conidios o estructuras conidiógenas, pero sus teleomorfos no son relacionados.

En los Hypocreales, los conidios en cadena no son comunes, pero en algunas especies de Fusarium (F. moniliforme), se producen. Mientras en los Eurotiales, esta situación es común. Los conidios de Gliocladium roseum, 'se unen en cadenas imbricadas, las cadenas de los conidióforos adyacentes pueden adherirse formando columnas visibles claramente a la lupa estereoscópica (debe diferenciarse de G.catenulatum, el cual forma cadenas conidiales que se tornan verdes después de 6-10 días. Normalmente los conidios formados en cadenas en los conidiomata del género Myrothecium, sólo se producen cuando se secan sus masas conidiales en el esporodoquio (Samuels \& Seifert 1987).

En los ciclos de vida de algunos hongos, la formación de 2 o hasta 5-6 tipos de conidios, o de distintos tipos de conidioforos, puede ser bastante frecuante en cultivo. Los Dermatofitos,son un buen ejemplo en Micología médica, como en los géneros comunes de Fusarium, Aureobasidium y muchos otros.

La formación de microconidios, es una característica común en especies de Fusarium, a veces estos se presentan como unica forma de conidiogénesis, sin formación de macroconidios (Gams \& Niremberg 1989), esta situación no debe catalogarse para la descripción de 2 géneros difrentes (concepto politético del género). Los distintos tipos de conidios pueden producirse en distintos tiempos generacionales, no todos al mismo tiempo; esta situación conlleva al estudio de todo el ciclo de vida fúngico (varias observaciones diferidas). Segun Booth (1979), algunas especies al crecer en sustratos efímeros, tienden a producir estructuras simples ya sea en la producción de los conidiomata, conidióforos y conidios, por la necesidad de un rápido recorrido de sus ciclos de vida , acorde a la naturaleza transitoria del sustrato.

\section{7.- PLEOANAMORFISMO (Sinanamorfos)}

Además de los procesos conidiogénicos clàsicos de tipo "Blàsticoy y Talico" que se produce en células diferenciadas especializadas, algunos gèneros de anamorfos son capaces de producir otros tipos de propágulos a partir de celulas conidiógenas diferenciadas o indiferenciadas de las hifas normales, las cuales pueden tener cierto valor taxonòmico a nivel de especie, como el caso específico de los "aleuroconidios, clamidoconidios y papuloconidios" .

Las diferencias entre los 2 primeros radica según Charmicael (1971),en que el aleuroconidio es un propágulo dehiscente mientras el segundo no lo es. El papuloconidio no es unverdadero conidio, sino un propàgulo particular producido por el gènero $\mathbf{P a p u}$ laspora, constituido por un bulbillo de cèlulas blandas que evolutivamente pueden haber derivado de ancestrales esclerocios o de inicios de pseudoascomas. La presencia de èstos u otros conidios producidos por una misma cepa (sinanamorfos), no debe dar origen a una nomenclatura binomial separada, aunque el CINB (Art.59) lo acepte. En la actualidad existen suficientes evidencias que permiten determinar que los distintos tipos de conidiogenesis no significan diferentes categorias. Un tipo deriva de otro (en la misma cepa), en concordancia a factores externos, de edad, nutricionales u otros que aùn no comprendemos claramente (ciclos de vida reducidos, Minter 1985). Si consideramos ademàs de los caracteres morfologicos, los quimiotaxonòmicos, veremos que 
la unidad de este individuo es evidente. Por lo expuesto, Hennebert 1991 ,concluye diciendo: "la diversidad morfologica, la plasticidad ontogènica de las formas y la unidad de los caracteres quimiotaxondmicos expresados por un solo pool de genes en la misma cepa, demuestra en forma clara la naturaleza artificial de las categorias monoanamorficas de los taxa y la necesidad de un concepto màs sintètico de la especie para llegar a una taxonomia màs natural".

Los ejemplos de sinanamorfos son comunes en los Hypocreales como en otros taxa, por ejemplo: Verticillium y Sepedonium, sinanamorfos de Hypomyces chrysospermum, Sibrina y Papulaspora, sinanamorfos de Hypomyces papulasporae, Verticillium y Gliocladium, sinanamorfos de Nectria ochroleuca, Scedosporium y Graphium, sinanamorfos de Pseudallescheria boidii.

\section{8.- EL ENFOQUE MODERNO}

A pesar que en la literatura las contribuciones aun enfatizan mayoritariamente la taxonomía morfológica tradicional, se ha observado en este altimo decenio un aumento notable de publicaciones que emplean la quimiotaxonomia, la ultraestructura, la serologia y la biologia molecular como nuevas herramientas taxondmicas, algunas de ellas aùn en franca experimentacion y ajuste, pero con enormes perspectivas futuras.

En la delimitaciòn de los gèneros u otra entidad taxonòmica inferior o superior, la microscopia electronica (TEM y SEM), ha permitido el estudio de caracteres ultraestructurales diversos, como la pared celular, los tipos de septos la conidiogènesis, elementos citoplasmatticos u otros. Por medio de su empleo se pueden establecer amplias interrelaciones entre los hongos que presentan pequeñas diferencias morfoldgicas, como asì en eventos meidticos y mitdticos (Boehout \& Linnemans 1982).

La serologia con sus estudios antigènicos, se considera cada vez màs ùtil para la identificacion ràpida de los hongos a nivel especifico o infraespecifico, ya sea en la micologia mèdica como en las afinidades geneticas de cepas parentales (Palonelli et al.1985,1988).

Los patrones totales de proteinas (isoenzimas) y su electroforesis, permiten la distincion entre especies u otros taxa infraespecificos. Esta metodologia se ha empleado ampliamente como una ayuda a la biologia y la sistemàtica, permitiendo diferenciar especies fitopatögenas de Puccinia (Burdon \& Marshall 1981), correlacionar en forma precisa grupos de compatibilidad vegetativa, patotipos y tipos electroforèticos en Fusarium (Bosland \& Williams 1987), en taxonomìa füngica y genètica de poblaciones en Asco y Basidiomycetes (Micales et al.1986, Riba et al.1987, Koch \& Kohler 1990, Hwang et al.1987, Hanson \& Wells 1991).

Los andisis de algunos componentes particulares de la cetula fùngica, tales como: polimeros y carbohidratos de pared, sistema de ubiquinonas (Coenzima Q), metabolitos secundarios (Micotoxinas principalmente), esteroles, acidos grasos, antraquinonas, test fisiologicos, etc., son metodos experimentales usados por muchos investigadores y en los centros especializados en la delimitación de gèneros y especies (CBS Newsletter 1988).

En la actualidad cabe destacar un notorio incremento de la biologia molecular como las tècnicas de anallisis de DNA, en especial las secuencias de RNA ribosomal (rRNA) de cadenas largas (ssrRNA o lsrRNA). Esta metodologia se emplea para señalar las relaciones filogenèticas de diversos organismos eucariontes o procarionres, como relacionar grupos de algas (Perasso et al. 1989, Bruns et al. 1989), o gèneros de levaduras (Guêho et al. 1990), o en bacterias (Woese 1987, Gouy \& Li 1989). Debido a que las moléculas de rRNA están compuestas por secuencias de segmentos altamente constantes, entremezclados con regiones de gran variabilidad, los genes ribosomales se presentan en las celulas fúngicas como grupos de unión únicos constituídos por elementos genéticos repetidos uno tras otro (en tandem), los cuales contienen pequeñas y largas subunidades. El nùmero de elementos que se repite varia dentro de las especies o entre ellas y en los hongos es en un nùmero cercano a las 100 copias; mecanismo a quien Dover (1982), puso el nombre de conducciòn molecular (molecular drive). El mecanismo que mantiene la homogeneidad en las secuencias entre las copias de genes de un individuo, es la conversion genica y el crossing-over desigual en la meiosis. Debido a la variada distribución fisica de los rRNA en los distintos grupos taxonòmicos y la diferente homogeneidad dentro de los individuos y de las especies, se asume la posibilidad de examinar las secuencias iguales (homogèneas) entre las cepas de una especie (Dover 1987). Esto hace posible la identificacion de especies por secuencias unicas de rRNA, tal como se ha comprobado en patrones de restriccion enzimaticos en rRNA o en fragmentos de largas secuencias (Peterson \& Logrieco 1991) u otros de separacion de proteinas aplicados a los àcidos nucleicos. Los mètodos filogenèticos directa o indirectamente apuntan sobre las relaciones del material genètico, màs que medir la expresion de sus determinantes geneticos (Brenner 1988).

\section{Conclusiones}

Como comenta Kendrick (1981), a pesar de las buenas intenciones de los taxdnomos en $\mathbf{H y}$. phomycetes, la clasificacion de sus taxa sigue siendo mayoritariamente artificial (aunque bastante pràctica), 
y de poco valor predictivo si se compara con los conceptos genèricos aplicados en los hongos teleomòrficos.

Es indudable que el micòlogo actual, netamente feneticista, no puede soportar por si solo, todo el peso de la informaciòn dispersa en otras disciplinas cientìficas que emplean a los hongos para sus estudios experimentales. Esta situaciòn modifica en mayor o menor grado el panorama taxonòmico actual aùn netamente morfo-anatòmico $y$ con un contenido especulativo en muchos casos, lo cual no es beneficioso para la sistemàtica del grupo, reflejandose en su inestable nomenclatura.

A pesar que los caracteres de la conidiogènesis, se reconocen como una herrramienta para el establecimiento de las relaciones naturales de los hongos anamòrficos (Cole y Samson 1979), despuès de casi 40 años de sobrevalorar la conidiogènesis y la cèlula conidiògena, aùn no hemos podido asociar en forma natural muchos grupos fùngicos a pesar de los buenos intentos personales de los especialistas, los cuales han sido aceptados o rechazados por la comunidad de micòlogos por la falta de criterios unànimes en la delimitaciòn morfològica de los taxa. En este sentido ha sido de gran ayuda el empleo del concepto genèrico politètico empleado por distintos taxònomos para evitar la dispersiòn y creaciòn de nuevos taxa pleoanamòrficos

Queda aùn mucho camino que recorrer, especialmente para aplicar toda la informaciòn disponible y evaluar la importancia de ciertos caracteres morfo-fisiològicos, la estrecha relaciòn entre comuni- dad - medio llevado a un efectivo enmascaramiento de las similitudes genèticas en estos hongos, las herramientas moleculares permitirán dilucidar en buena medida estas interrogantes en un futuro cercano. La expansiòn sustancial en el uso de mètodos filogenèticos y su aplicaciòn en la identificaciòn de diversos microorganismos, merece ser examinada seriamente, por su impacto sobre la sistemática y la nomenclatura, pero tambièn por su aporte a nuevos conocimientos biològicos, clìnicos, o por la utilidad pràctica que involucra identificar nuevos taxa.

Los resultados experimentales aùn incompletos, pueden sobredimensionar la informaciòn obienida. Las secuencias relacionadas de àcidos ruclèicos pueden tener un $5 \%$ o menos de divergencia; consecuentemente estas relaciones son consideradas similitudes màs que identidades y se recomienda que las caracterìsticas fenèticas deben ser concordantes con la definiciòn filogenètica de especie, la cual no debe ser considerada la base primaria para la especiaciòn.

Estamos en la alborada de una nueva taxonomìa del grupo, donde muchos de los géneros descritos por conveniencia en el pasado, sin una clara relaciòn genealògica, podràn tener la jerarquìa que la evoluciòn les asignò. Las controversias entre los taxònomos profesionales no dejaràn de existir, seguramente se acentuaràn con los futuros aportes, pero debemos seguir el camino con optimismo renovador, quizàs esto ùitimo sea el meior tributo a nuestra paciencia.

\section{Agradecimientos.}

Se agradecen los comentarios y las ùtiles sugerencias efectuadas por el Prof.Dr. Giuseppe Caretta en la confecciòn de este manuscrito.

\section{REFERENCIAS}

Arambarri,A.M \& Cabello,M.N (1989). A numerical taxonomy study of some phialidic genera of Hyphomycetes; cluster analisis Mycotaxon $34: 679-696$

Boekhout,T.\& Linnemans,W.A.N (1981). Ultrastructure of mito sis in Rhodosporidium toruloides Stud. Mycol. 22 : 23-38

Booth,C. (1971). The genus Fusarium. C.M.I. Kew, Surrey. England

(1979). Do you belive in genera ?. Trans.Br.Mycol.Soc $71: 1-9$

Bosland,P.W \& Williams,P.H. (1987). An evolution of Fusarium oxysporum from crucifers based on pathogenicity, isozyme polimorphism,vegetative compatibility, and geographic origin . Can. J. Bot. 65 : 2060-2073

Brasier,C.M \& Rayner,A.D.M. (1987). Whither terminology be low the species level in the fungi ?. In : Evolutionary biology of the fungi (Eds.) Rayner.A.D.M. et al. Cambriog University Press. Cambridge pp 381-288

Bridge, P.D., Hawksworth, D.L_,Kozakiewicz,Z,, Onions, A.H.S., Paterson,R.R.M., Sackin,M.J. \& Sneath,P.H.A (1989). A reappreisal of the terverticillate Penicillia, using biochemi cal, physiological and morphological features.I. Numerical taxonomy. J.Gen.Microb. 135 : 2941-2966 
Dover,G.A. (1982). Molecular drive. a cohesive mode of specie evolution. Nature 299 : 111-117

(1987). DNA turnover and the molecular clock. J. Mol. Evol. 26 : $47-58$

Frisvald, J.C., Filtenborg,O, Samson,RA. \& Stolk_A.C. (1990). Chemotaxonomy of the genus Talaromyces. Antonic van Leewenhoek 57 : 179-189

Gams, W. (1971). Cephalosporium artige Schimmelpilze (Hyphomycetes). Gustav Fischer, Vertag. Stuttgart

(1973). Phialides with solitary conidia ? Remarks on conidium ontogeny in some Hyphomycetes. Persoonia 7 : 161-169

\& McGinnis,R.M. (1983). Phialemontum, a new anamorph genus intermediate between Phialophora and Acremoniam. Mycologia $75: 977-987$

\& Niremberg, H.I. (1989). A Contribution to the generic definition of Fusarium. Mycotaxon 35 : 407-416

Gouy,M. \& Li,Wen-Hsiung (1989). Phylogenetic analisis based on rRNA sequences supports the archaebacterial rather than the eocyte tree. Nature $339: 145-147$

Guêho,E., Kurtzman,C.P. \& Peterson,S.W. (1990). Evolutionary affinities of heterobasidiomycetous yeast estimated from $18 \mathrm{~S}$ and $22 \mathrm{~S}$ ribosomal RNA sequence divergence. Syst.Appl.Microbiol. 13 : 230-236

Hanson,L.C. \& Wells,K. (1991). Characterization of three Tremella species by isozyme analisis. Mycologya $83: 446-454$

Hennebert,G.L. (1991). Art. 59 and the problem with pleoanamorphic fungi. Mycotaxon $40: 479-496$

Holubovà-Jechovà, V. (1990). Problems in the taxonomy of the dematiaceous Hyphomycetes. Stud. Mycol. C.B.S. 32: 41-48

Hughes, S. J. (1953). Conidiophores, conidia and classification. Can J. Bot. $31: 577-659$

(1958). Revisiones Hyphomycetum aliquot cum appendice de nominibus rejiciendis. Can.J.Bot. $36: 727-836$

Hwang,B.K., Yun,J.H. \& Kim,Zs. (1987). Geographic variation of esterase isozyme in population of Alternaria mali on apple leaves. J.Phytophath. 119 225-23!

Kirk, P. M. \& Sutton, B. C. (1985). Areassessement of the anamorph genus Chaetopsina (Hyphomycetes). Trans. Br. Mycol. Soc. 85 : $709-718$

Koch, G. \& Kohler, N. (1990). Isozyme variation and genetic đistance of Erysiphe graminis de formae speciales. J. Phytopath. $129: 89-101$
Micales,JA,Bonde,M.R. \& Peterson,G.L. (1986). The use of isozyme analisis in fungal taxonomy and genetics Myco $\operatorname{tax}$ on $27: \mathbf{4 0 5 - 4 4 9}$

Minter,D.W. (1985). A reappraisal of the relationships between Arthrinium and other Hyphomycetes. Proc. Indian. Acad. Sci. (Plant Sci) $94:$ 281- 308

Kirk,P.M. \& Sutton,B.C. (1982). Holoblastic phialides. Trans. Br. Mycol. Soc. 79 : 75-93

Sutton, B.C. \& Brady,B.I. (1983). What are phialides anway? Trans. Br. Mycol. Soc. $81: 109-120$

(1987). What is happening in Ascomycete classification? The mycologist $2: 55-63$

Mishler,B.D. (1991) An overview of competing concepts of species and speciation Newsletter M.S.of Amer. $42: 27$

Monte, Em, Bridg3e,P.D. \& Sutton ,B.C. (1990). Physiological and biochemical studies in Coclomycetes Phoma. Stud. Mycol 32 : $21-28$

Morgan-Jones, C. \& Gams, W. (1982)- Notes on Hyphomycetes XII. An endophyte of Festuca arundinacea and the anamorph of Epichloe typhina, new taxa in one of two new sectionof Acremonium. Mycotaxon 15 : 311-318

\& Jacobsen,B.J.(1988). Notes on Hyphomycetes LVII. Some dematiaceous taxa, including two undescribed species of Cladosporium, associated with biodeterioration of carpet. plaster and wallpaper. Mycotaxon $32 \cdot 223-236$

Mugnai, Lo, Bridge, P. D. \& Evans, H. C. (1989). A Chemosistematic evaluation of the genus Beauveria. Mycol res. 92 199-209

Okoshi,s. \& Hasegawa,A. (1966). Abortive cleistohecia of Tri. chophyton mentagrophytes from dog and rat ringworm. Jap. J. Med, Mycol. 7: 31-35

Palonelli, L, Castagnola, M., D’Urso, C. \& Morace, G. (1985). Serological approaches for identification of Aspergillus and Penicillium species. In: Advances in Penicillium and Aspergillus systematics. (Eds.) Samson. R.A. \& Pitt. J I Plenum Press.New York and London pp. 267-279

.Dettoru, G. Rosa, R. Castagnola, M. \& Schipper. M.A.A. (1988) Antigenic studies on Rhizopus microsporus, Rh. rhizopodiformis, progeny and intermediates (Rh. chinensis). Antonie van Leeuwenhoek $54: 5-18$

Pascoe,I.G. (1990a). Fusarium morphology I. Identification and characterization of a third conidial type, the mesoconidium. Mycotaxon $37: 121-160$

(1990b). Fusarium morphology II. Experiments on growing conditions and dispersal of mesoconidia. Mycotaxon 37: 161-172 
Sutton, B.C. \& Brady,B.L. (1983). What are phialides anway ? Trans. Br. Mycol. Soc. $81: 109-120$

(1987). What is happening in Ascomycete classification? The mycologist $2: 55-63$

Mishler,B.D. (1991) An overview of competing concepts of species and speciation Newsletter M.S.of Amer. 42: 27

Monte, E, Bridg3e,P.D. \& Sutton, B.C. (1990). Physiological and biochemical studies in Coelomycetes Phoma. Stud. Mycol. 32: 21-28

Morgan-Jones, C. \& Gams, W. (1982)- Notes on Hyphomycetes. XLI. An endophyte of Festece aruadinacea and the anamorph of Epichloe typhina, new taxa in one of two new section of Acremoniam. Mycotaxon 15 : 311-318

\& Jacobsen,B.J.(1988). Notes on Hyphomycetes LVII. Some dematiaceous taxa, including two undescribed species of Cladosporium, associated with biodeterioration of carpet, plaster and wallpaper. Mycotaxon 32 : 223-236

Mugnai, Lo, Bridge, P. D. \& Evans, H. C. (1989). A Chemosistematic evaluation of the genus Beauveria. Mycol res. 92 : 199-209

Okoshi,s. \& Hasegawa,A. (1966). Abortive cleistohecia of Trichophyton mentagrophytes from dog and rat ringworm. Jap. J. Med, Mycol. 7 : 31-35

Palonelli, L, Castagnola, M. D'Urso, C. \& Morace, G. (1985). Serological approaches for identification of Aspergillus and Penicillium species. In: Advances in Penicillium and Aspergillus systematics. (Eds.) Samson, R.A. \& Pitt, J.I. Plenum Press,New York and London pp. 267-279

Dettore, G., Rosa, R., Castagnola, M. \& Schipper, M.A.A. (1988). Antigenic studies on Rhizopus microsporus, $\mathbf{R h}$ rhizopodiformis, progeny and intermediates ( $\mathbf{R h}$. chinensis). Antonic van Leeuwenhock 54 : 5-18

Pascoe,I.G. (1990a). Fusarium morphology I. Identification and characterization of a third conidial type, the mesoconidium. Mycotaxon 37 : 121-160

(19906). Fusarium morphology II. Experiments on growing conditions and dispersal of mesoconidia. Myco taxon $37: 161-172$

Perasso, R, Baroin, An, Qu, L.H., Bachellerie, J.P. \& Adoutle, A. (1989). Origin of the algac. Nature $339: 142-144$

Peterson,S.W. \& Logrieco, A. (1991). Ribosomal RNA sequence variation among interfertile strains of some Gibberells species. Mycologia 83 : $397-402$

Pitt,J.I. (1979). The genus Penicillium and its teleomorphic states Eupenicillium and Talaromyces. Academic Press, London

\& Hocking,D.L. (1985). The naming of chemical variants in Penicillium and Aspergillus. In : Advances in Penicil-
Hom and Aspergillus systematics. (Eds.) Samson, R.A. \& Pitt, J.I. Plenum Press N.York, London pp. $89-91$

Samson, R.A. (1989). Approaches to Penicillium and Aspergillus systematics. Stud. Mycol. 32 : 77-90

Prasil,K. \& De Hoog,G.S. (1988). Variability in Cladosporium herbarum. Trans. Br. Mycol. Soc. 90 : $49-54$

Riba, G, Soarez, G. F, Samson, R. A, Onillon, J. \& Caudal, A. (1987). Isozyme analysis of isolates of the entomogenous fungi Tolypoeledium cylindrosporum and T. extinguens (Deuteromycotina, Hyphomycetes). J. Invert. Path. 48 : 362-367

Romero,1A. \& Minter,D.W. (1988). Fluorescence microscopy : An aid to the elucidation of Ascomycete structure. Trans. Br. Mycol. Soc. $90: 457-470$

Rossman, A. Y. (1983). The phragmosporus species of Nectria. Mycol Pap. 150: 1-164

Saito,Y, Tanaka,S. \& Watanabe,S. (1991). Ultrastructure of the pseudo-cleistothecia produced by the Trichophyton men. tagrophytes complex. J. Med.Vet. Mycol. 29 : 225-233

Samson,R.A. \& Pitt,J.I. (Eds.) Modern concepts in Penicillium and Aspergillus. Plenum Press,N.York and London.

Samuels, GJ. (1976). A revisión of the fungi formerly classified as Nectria Subgenus Hyphonectria. Mem.N.York Bot. Gard. $26: 1-126$

(1985). Four news species of Nectria and their Chaetopsina anamorph. Mycotaxon 22 : 13-32

\& Seifert,K.A. (1987). Taxonomic implications of variation among Hypocrealean anamorph. In: Pleoanamorphic fungi: The diversity and its taxonomic implictions. (Ed. J. Sugiyama) Kodansha Ltd. Tokyo and Elsevier Amsterdam pp. 29-56

Seifert, K.A. (1985). A monograph of Stilbella and some allied Hyphomycetes. Stud. Mycol. C.B.S. 27 : 1-235

\& Samson, R.A. (1985). The genus acremonium and the synnematous Penicillia in Advances in Penicillium and Aspergillus Systematics (Eds. Samson, R.A. \& Pitt, J.J.) Plenum Publishers N. York, pp. 143-154.

\& Okada, G. (1990). Taxonomic implications of conidiomatal anatomy in synnematous Hyphomycetes. Stud. Mycol. 32 : 29-40.

Stockdale, P. (1961). A perfect state of Mierosporum gypseum (Bodin) Guiart et Grigorakis. Sabouraudia 1 : 41-48.

Van der, Aa. J; Noordeloos, M. E. \& De Gruyter, J. (1990). Species concepts in some larger gencra of the Coelomyces. Studies in Mycology 32 : 3-19

Woese, C.R. (1987). Bacterial evolution. Microbiol. Rev.51 : 221 . 271. 